\title{
GROUPS OF FINITE WEIGHT
}

\author{
A. H. RHEMTULLA ${ }^{1}$
}

\begin{abstract}
If $N$ is a group and $E$ is a group of operators on $N$ then write $d_{E}(N)$ for the minimum number of elements needed to generate $N$ as an $E$-group. It is shown that if $N$ is a normal subgroup of $E$ and $E$ acts on $N$ by conjugation, then $d_{E}(N)=d_{E}\left(N / N^{\prime}\right)$ if $d_{E}(N)$ is finite and there does not exist an infinite descending series of $E$-normal subgroups $N^{\prime}=C_{0}>C_{1}>\cdots$ with each $C_{i} / C_{i+1}$ perfect. Both these conditions are, in general, necessary.
\end{abstract}

If $N$ is a group and $E$ is a group of operators $N$ then we shall write $d_{E}(N)$ for the minimum number of elements needed to generate $N$ as an $E$-group. We shall call this number the $E$-weight of $N$ and use the convention $d_{E}(N)=1$ if $N$ is the trivial group. The main result of this note is to prove the following result referred to by $K$. W. Gruenberg in [2, p. 74].

THEOREM. If $N$ is a normal subgroup of a group $E$ and $E$ acts on $N$ by conjugation, then $d_{E}(N)=d_{E}\left(N / N^{\prime}\right)$ provided $d_{E}(N)$ is finite and $N$ has the following property.

(*) There does not exist an infinite descending series of $E$-subgroups $N^{\prime}=C_{0}>C_{1}$ $>\cdots$ with each $C_{i} / C_{i+1}$ perfect.

We have used $N^{\prime}$ to denote the commutator subgroup of $N$. A group $G$ is perfect if $G=G^{\prime}$. Note that if the lattice of normal subgroups of $E$ contaned in $N^{\prime}$ satisfies the minimum condition then the property (*) is trivially satisfied. Thus as a corollary we obtain the following result of P. Kutzko [5].

If $G$ is a group such that $d_{G}(G)$ is finite and the lattice of normal subgroups of $G$ which are contained in $G^{\prime}$ satisfies the minimum condition then $d_{G}(G)=$ $d_{G}\left(G / G^{\prime}\right)$.

It is important to point out that the above result of Kutzko was obtained by $\mathbf{R}$. Baer in [1]. The purpose of this note is to supply a proof of a result mentioned in the literature.

Proof of THE THeORem. Suppose the result is false. Let $C_{0}=N^{\prime}$. Let $d_{E}\left(N / C_{0}\right)$ $=k$. Then there exist $a_{1}, \ldots, a_{k}$ in $N$ such that $A_{0}=\left\langle a_{1}, \ldots, a_{k}\right\rangle^{E}$ satisfies $A_{0} C_{0}=N$. If $A_{0} \geqslant C_{0}$ then $A_{0}=N$ and we have the required contradiction. Let $B_{0}=A_{0} \cap C_{0}$ so that $B_{0}<C_{0}$. Note that since $C_{0} \leqslant N^{\prime}, C_{0} / B_{0}$ is perfect. Also since $d_{E}(N)$ is finite, so is $d_{E}\left(N / B_{0}\right)$. Thus there exists a normal subgroup $C_{1}$ of $E$ such that $B_{0} \leqslant C_{1}<C_{0}$ and $d_{E}\left(C_{0} / C_{1}\right)=1$. Now $C_{0} / C_{1}$ is perfect and $C_{0}=$ $\left\langle C_{1}, x\right\rangle^{E}$ for some $x$ in $C_{0}$.

Received by the editors March 31, 1980.

AMS (MOS) subject classifications (1970). Primary 20F05; Secondary $20 \mathrm{~F} 15$.

${ }^{1}$ Research partially supported by a grant from the Natural Sciences and Engineering Research Council, Canada. 
Let $A_{1}=\left\langle a_{1} x, a_{2}, \ldots, a_{k}\right\rangle^{E}$. Since $\left[a_{1}, C_{0}\right]<C_{0} \cap A_{0}=B_{0}<C_{1}, C_{1}\left[a_{1} x, C_{0}\right]^{E}$ $=C_{1}\left[x, C_{0}\right]^{E}$ and $C_{0} / C_{1}\left[x, C_{0}\right]^{E}$ is cyclic. But $C_{0} / C_{1}$ is perfect. Hence $C_{1}\left[a_{1} x, C_{0}\right]^{E}=C_{0}$ and $A_{1} C_{1}=N$. Note that $d_{E}\left(N / C_{1}\right)=k$. Let $B_{1}=A_{1} \cap C_{1}$ and repeat the above argument with $A_{1}, B_{1}, C_{1}$ replacing $A_{0}, B_{0}, C_{0}$ respectively. This process yields a sequence of $N^{\prime}=C_{0}>C_{1}>\cdots$ where $C_{i}$ are normal in $E$ and each $C_{i} / C_{i+1}$ is perfect, contradicting property (*). This completes the proof.

REMARK 1. The condition that $d_{E}(N)$ be finite is, in general, necessary in the above theorem. Heineken and Wilson's example in [3] shows this. They constructed a countable, locally solvable, perfect group $G$ satisfying the minimal condition on normal subgroups and at the same time $G$ is isomorphic to all its nontrivial homomorphic images. Since $G$ is perfect, $d_{G}\left(G / G^{\prime}\right)=1$. By the above theorem $d_{G}(G)=1$ or $\infty$. If $G=\left\langle g^{G}\right\rangle$ for some $g$ in $G$, then look at $G / M$ where $M$ is a maximal normal subgroup of $G$ subject to not containing $g$. Then every normal subgroup of $G / M$ contains $M g$. Thus $G / M$ is simple. But $G / M$ is locally solvable and, as is well known, a locally solvable simple group is trivial. Thus $d_{G}(G)=\infty$.

Remark 2. The condition (*) in the theorem is, in general, necessary. M. A. Kervaire has shown in [4] that the free product $F$ of $G$ and $C_{\infty}$ where $G=\langle\alpha, \beta$; $\alpha^{2}=\beta^{2}=\left(\alpha^{-1} \beta\right)^{5}>$ has the property that $F / F^{\prime} \cong C_{\infty}$, the infinite cyclic group, so that $d_{F}\left(F / F^{\prime}\right)=1$. But $d_{F}(F)>1$.

\section{REFERENCES}

1. R. Baer, Der reduzierte Rang einer Gruppe, J. Reine Angew. Math. 214/215 (1964), 146-173.

2. K. W. Gruenberg, Free abelianised extensions of finite groups, Homological Group Theory, C. T. C. Wall (Ed.), London Math. Soc. Lecture Notes, no. 36, London Math. Soc., 1979, pp. 71-104.

3. H. Heineken and J. S. Wilson, Locally soluble groups with Min-n, J. Austral. Math. Soc. 17 (1974), 305-318.

4. M. A. Kervaire, On higher dimensional knots, Differential and Combinatorial Topology (A Sympos. in Honour of Marston Morse), Princeton Univ. Press, Princeton, N. J., 1965, pp. 105-109.

5. P. Kutzko, On groups of finite weight, Proc. Amer. Math. Soc. 55 (1976), 279-280.

Department of Mathematics, University of Alberta, Edmonton, Alberta, Canada T6G 2G1 